\title{
Nonlinear Discontinuous Dynamics Averaging and PWM Based Sliding Control of Solenoid-Valve Pneumatic Actuators
}

\author{
Sean Hodgson ${ }^{1}$, Mahdi Tavakoli ${ }^{1}$, IEEE Member, Minh Tu Pham ${ }^{2}$, IEEE Member, Arnaud Leleve ${ }^{2}$, IEEE Member \\ ${ }^{1}$ Department of Electrical and Computer Engineering, University of Alberta, Edmonton, AB T6G2V4, Canada \\ ${ }^{2}$ Laboratoire Ampère, UMR CNRS 5005, Université de Lyon, INSA-LYON, F-69621 Villeurbanne Cedex, France
}

\begin{abstract}
A pneumatic actuator with solenoid valves is a discontinuous-input system because each valve can be either in on or off state. For such an actuator, this paper proposes a slidingmode control scheme that is based on an averaged continuousinput model of the discontinuous-input open-loop system. The averaged model is obtained from the nonlinear dynamics of the open-loop system undergoing pulse-width-modulation (PWM) at the input (i.e., valve open/close action). The PWM duty cycle will be regarded as a continuous input to the proposed averaged model, and thus generated by the proposed slidingmode controller.

For the sliding control design, we note that a pneumatic actuator has two chambers with a total of four on/off valves. Thus, there are sixteen possible combinations for valves' switching. Seven of these sixteen operating "modes" are considered both functional and unique. The proposed sliding control utilizes and switches between these seven modes of the open-loop system in order to select the ones with necessary and sufficient amounts of drive energy. In comparing the new 7-mode controller to previous controllers, we will demonstrate reductions in the position tracking error and the number of switches made by the actuator's on/off valves. The proposed control scheme is used in both position control of a pneumatic cylinder and bilateral control of a one degree of freedom teleoperation system. Experimental results are presented to validate our theoretical findings.
\end{abstract}

Index Terms-Pneumatic actuator, on/off solenoid valve, PWM, sliding-mode control, position tracking, force tracking, teleoperation

\section{NOMENCLATURE}

$P_{P}, P_{N}$

Chamber pressure $(\mathrm{Pa})$.

$V_{P}, V_{N}$

Chamber volumes $\left(\mathrm{m}^{3}\right)$.

$Q_{P}, Q_{N}$

Input mass flow rates $\left(\mathrm{kg} . \mathrm{s}^{-1}\right)$.

$T_{P}, T_{N}, T$ Chamber temperature (K).

$A_{P}, A_{N}, A$ Piston cylinder areas $\left(\mathrm{m}^{2}\right)$.

$y$

$y_{d}$

$y_{m} / y_{s}$

$\tau_{\text {Ext }}$

$\tau_{h} / \tau_{e}$

$\tau$

$\tau_{d}$

$\tau_{S t}$

$k$

$r$

l

$U_{X}$
$P_{S}$

$P_{E}$

$M$

Source pressure $(\mathrm{Pa})$.

Exhaust pressure $(\mathrm{Pa})$.

Total mass of the load and the piston $(\mathrm{kg})$.

\section{INTRODUCTION}

Recent developments in robotics and telerobotics have allowed working in environments that are normally difficult or hazardous for the human hand. Telerobotic systems have been developed for applications ranging from surgery to micromanipulation to space exploration [1], [2], [3], [4], [5], [6].

In this study, we investigate the control of pneumatic actuators in single actuator and a 1-DOF telerobot applications. Pneumatic actuators are widely used in many different industries and offer many advantages such as low cost, good power/weight ratio, cleanliness, and safety [7], [8], [9]. Moreover, they permit to set actuators' compliance up more easily than with electric ones by controlling the amount of air inside both chambers [10], [11]. However, they suffer from drawbacks including friction and variation of the actuator dynamics to load and piston position along the cylinder stroke [12] and accurate position control of such actuators is difficult due to the nonlinear dynamics [13]. Despite these difficulties, this kind of systems could be found in medical robotics applications for needle insertion, MRI compliant telerobots (where electric actuators are banned) [14], and exoskeletons [15] for rehabilitation purpose.

A significant amount of high quality research has been accomplished in this field of research. Ben-Dov et al. implemented a force controlled actuator based on voice-coil flapper valves and low-friction cylinders [16]. Whereas, Varseveld et al. used a novel PWM algorithm on on/off solenoid-valves to control the position of a pneumatic actuator [17]. Takaiwa et al. implemented a haptic interface without a force/momentum sensor employing a pneumatic parallel manipulator to realize "information transfer by means of contact" [18]. Shen et al. also created a haptic interface which implemented a pneumatically actuated impedence to simulate stiffness [19]. Ying et al. developed an arm-exoskeleton with pneumatically driven force-feedback systems employing on/off valves and fuzzy logic controllers [15]. All of these works demonstrated how effective model-based control can be for implementing a pneumatically actuated system. This paper employs sliding mode control in order to guarantee the parametric robustness of the pneumatic actuator model and also the friction and load variations. 


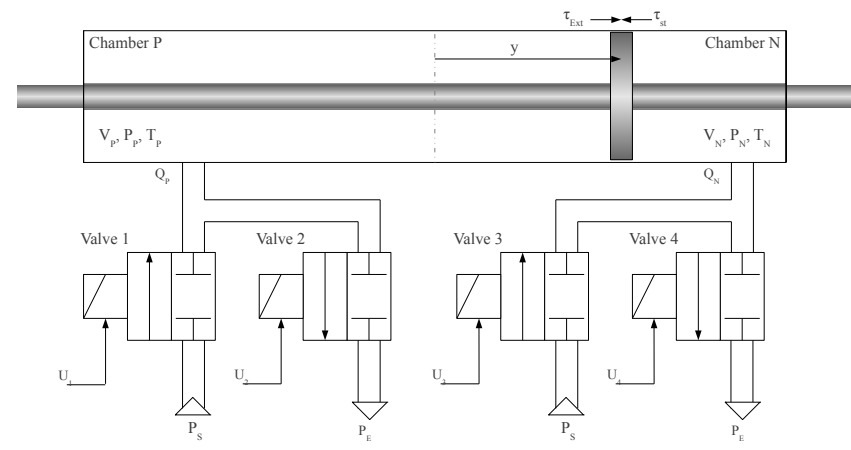

Fig. 1. Single pneumatic actuator with 4 on/off solenoid valves.

For excitation, some pneumatic systems are equipped with on/off solenoid valves. When compared to servo-valves, solenoid-valves can provide a lower-cost alternative for controlling the mass flow rate. In a medium or large series manufacturing, this criterion is important. A PWM input with a high switching frequency approximates a continuous input whose magnitude is determined by the PWM duty cycle. This is advantageous because it allows us to provide to a solenoid switching valve what is effectively a continuous input. On/off control implies controlling the on/off voltage of the solenoid valves. PWM control implies controlling the duty-cycle of the solenoid valves. Utilizing a pure on/off-control provided by the solenoid-valves will make precise control of the piston position difficult.

If the nonlinear dynamics of a pneumatic system can be approximated as an equivalent linear system, then linear controllers can be designed [7]. It is preferable to design pneumatic controllers that take into account the actuator's nonlinear dynamics. PWM controllers have been developed in the past to control the position of solenoid-valve pneumatic actuators [20], [21], [22], [23].

It is possible to employ nonlinear sliding-mode control for solenoid-valve systems [24], [25]. Sliding-mode control alters the dynamics of a system by the application of a highfrequency switching control [26], [27]. Sliding-mode control is parametrically very robust and can account for dynamic uncertainties as well as nonlinearities of a system. In [28], nonlinear model averaging is combined with PWM and sliding-mode control to control a solenoid-valve pneumatic actuator.

\section{Context and Contributions}

In this paper, we consider a 1-DOF pneumatic actuator composed of two chambers, each one connected to two solenoid valves, as shown in Figure 1. As detailed in [29], this configuration allows to get nine discrete "modes" at any given time.

In [28], only three of these discrete modes are considered for a two-chamber solenoid-valve actuator (similar to the one shown in Figure 1): "Push and Pull", "Pull and Push" and "Close and Close" (both chambers' valves closed). In [30], we proposed a switching control law which was validated on an electropneumatic positioning system. This controller enabled to choose the best mode, amongst three and seven available modes, to apply at each sampling period. In [31], a hybrid predictive controller using two additional operating modes was utilised with different teleoperation schemes. We showed that employing extra modes facilitates the use of more appropriate amounts of drive energy for a good position control. Indeed the additional modes allow more refined increments to the mass flow rate that can be provided to each chamber. For instance, these additional modes could deliver a force smaller than the pull-and-open mode in order to avoid overshoots and to reduce the steady state error around the desired value. They also reduce the valve switching activity by controlling each mode change in each PWM period. In the current paper, we develop, an averaged model (for three and seven modes). Unlike our previous works, this model allows to design a sliding mode controller, where an equivalent control law could be used on the sliding surface. We also show some proof of stability of the proposed controller and we apply it in a teleoperation context.

The organization of this paper is as follows. The discrete input model of the actuator is found in Section II. A nonlinear averaged continuous-input model of the open-loop actuator is obtained in Section III. The averaged model is obtained from the nonlinear dynamics of the open-loop system undergoing pulse-width modulation at the input (i.e., valve open/close action). A sliding-mode position control for a single pneumatic actuator is proposed in Section IV. The experimental results validating the proposed controller is shown in Section V. Finally, the concluding remarks are presented in Section VI.

\section{Discrete-InPut Model of the Open-Loop ACTUATOR}

As shown in [30], it is possible to write the dynamics of the open-loop pneumatic actuator in a discrete input form. Assuming that the temperature variation in chambers is negligible with respect to the supply temperature (thus $T_{P}=T_{N}=T$ ), the dynamics of the actuator are obtained as

$$
\begin{array}{r}
\dddot{y}=f+\frac{k r T}{M}\left(\frac{Q_{P}}{l / 2+y}-\frac{Q_{N}}{l / 2-y}\right)+\frac{\dot{\tau}_{E x t}}{M} \\
f=\frac{-b_{V}}{M} \ddot{y}-\frac{k}{M}\left(\frac{A_{P} P_{P}}{l / 2+y}+\frac{A_{N} P_{N}}{l / 2-y}\right) \dot{y}
\end{array}
$$

Since each chamber can be in one of the three states of pressurizing, venting or closed, there are a total of nine discrete modes for the two-chamber actuator [30]. These modes are shown in Table I.

TABLE I

Nine Discrete Modes of The Open-Loop Actuator

\begin{tabular}{cccccccc|cc}
\hline \hline & $M_{1}$ & $M_{2}$ & $M_{3}$ & $M_{4}$ & $M_{5}$ & $M_{6}$ & $M_{7}$ & $M_{8}$ & $M_{9}$ \\
\hline$U_{1}$ & 0 & 1 & 0 & 0 & 0 & 1 & 0 & 0 & 1 \\
$U_{2}$ & 0 & 0 & 1 & 0 & 0 & 0 & 1 & 1 & 0 \\
$U_{3}$ & 0 & 0 & 0 & 0 & 1 & 0 & 1 & 0 & 1 \\
$U_{4}$ & 0 & 0 & 0 & 1 & 0 & 1 & 0 & 1 & 0 \\
\hline \hline
\end{tabular}

For each of these nine discrete modes:

$$
\dddot{y}= \begin{cases}f+\frac{\dot{\tau}_{E x t}}{M} & , \text { mode } M_{1} \\ f+(-1)^{j} b_{j}+\frac{\dot{\tau}_{E x t}}{M} & , \text { mode } M_{j} \neq M_{1}\end{cases}
$$


where the integer $j$ ranges from 2 to 9 and

$$
\begin{aligned}
b_{2}=\frac{k r T}{M} \frac{Q\left(P_{S}, P_{P}\right)}{(l / 2+y)} & b_{3} & =\frac{k r T}{M} \frac{Q\left(P_{P}, P_{E}\right)}{(l / 2+y)} \\
b_{4}=\frac{k r T}{M} \frac{Q\left(P_{N}, P_{E}\right)}{(l / 2-y)} & b_{5} & =\frac{k r T}{M} \frac{Q\left(P_{S}, P_{N}\right)}{(l / 2-y)} \\
b_{6}=b_{2}+b_{4} & b_{7} & =b_{5}+b_{3} \\
b_{8}=b_{4}-b_{3} & b_{9} & =b_{5}-b_{2}
\end{aligned}
$$

Note that because $P_{E} \leq P_{P} \leq P_{S}, P_{E} \leq P_{N} \leq P_{S}$, $-l / 2 \leq y \leq l / 2$, and mass flow rates are non-negative, functions $b_{2}$ through $b_{7}$ are all positive or equal to zero. Also, $b_{8}$ and $b_{9}$ are approximately equal to 0 and can be positive or negative.

If we focus on the $U_{i}$ signals, we observe that $M_{4}\left(U_{4}\right.$ on) and $M_{6}\left(U_{1}+U_{4}\right.$ on) both open valve 4 . If we need an actuation located between $\gamma_{4}$ and 1, we obtain, for instance, $d_{4}=0.4$ and $d_{6}=0.6$. We then activate $M_{4}$ mode during $40 \%$ of the PWM period and then $M_{6}$ during the remaining $60 \%$. As in both $M_{4}$ and $M_{6}$ modes, valve 4 is to be open, $U_{4}$ signal remains 1 during the whole PWM period (so no switching of $U_{4}$ during this period). Meanwhile, $U_{1}$ is powered on during $60 \%$ of this period. This control law necessitates one switch per PWM period while 3 mode one necessitates 2 switches (from $M_{1}$ to $M_{6}, U_{1}$ and $U_{4}$ have to be switched on). This is the same case in low actuation regions; for example when $u \in\left[0 ; \gamma_{4}\right]$, the valves alternatively switch between modes $M_{1}$ and $M_{4}$ where only the state of $U_{4}$ differs. In 3 mode case, the system would have also switched $U_{1}$ and $U_{4}$, so 2 switches inside the PWM period. By managing by ourself the PWM switching, we manage to reduce the number of switchings.

Thus, the modeling and control analysis from this point forward will focus on the modes $M_{1}$ to $M_{7}$.

In the next section, we will obtain an averaged continuousinput equivalent for the above discontinuous-input open-loop model. This averaged model is obtained from the nonlinear dynamics of the open-loop system undergoing pulse-width modulation at the input (i.e., valve open/close action). The PWM duty cycle will acts as the continuous input to the proposed averaged model.

\section{Averaged Continuous-InPut Model of the OPEN-LOOP ACTUATOR}

In [30], [31] the controllers could be seen as discretetime event controllers. Indeed the control laws used in these papers were directly the input voltages of the different solenoid valves. Contrary to these strategies, we propose in the current paper to design a time continuous controller based on an averaged model the different available modes of the system. The new input of such a model is the duty cycle, which is a time continuous signal. This transformation from a discrete to a continuous input allows to use the framework of sliding mode control theory in order to design and to prove the stability of a switching controller.

Let us consider a general dynamic system that may operate in one of $p$ distinct modes at any given time. Within a PWM "period", the system can switch between modes 1 through $p$, perhaps due to the variations in the input provided to the system. Assume that switching between the modes 1 through $p$ happens according to the modal duty cycle (duration) $d_{i}$. Then,

$$
D=\left[d_{1}, d_{2}, \ldots, d_{p}\right]^{T}
$$

gives the PWM period. The total duration of the modal duty cycles must equal the total PWM period, which is usually normalized to unity, i.e., $\|D\|_{1}=1$. If the system has dynamics $y^{(n)}=f_{i}$ when in mode $i$, where $n$ is the system order, and we collect the system dynamics for the $p$ modes in the vector

$$
F=\left[f_{1}, f_{2}, \ldots, f_{p}\right]^{T}
$$

then a time-averaged model $y_{a}^{(n)}$ of the system dynamics $y^{(n)}$ can be given by [28]

$$
y_{a}^{(n)}=F^{T} D
$$

Therefore, if we can implement the PWM period as a function of a single continuous input $u$ we obtain two things: a duty cycle mapping for the $p$-mode system that can be used to operate the PWM, and a time-averaged dynamic model which can be utilized to design the sliding control.

\section{A. Duty Cycle Mapping for the 3-Mode System}

Shen et al. have applied such a nonlinear model averaging to a 3-mode pneumatic actuator [28]. We will first derive that averaged model and then extend the method to the case of a 7-mode actuator model. Using (3) for a single pneumatic actuator without external disturbances, we have

$$
f_{i}= \begin{cases}f & , \mathrm{i}=1 \\ f+(-1)^{i} b_{i} & , \text { otherwise }\end{cases}
$$

for $i \in\{1,6,7\}$ because, as discussed previously, these are the three modes corresponding to "Close and Close" (mode 1), "Push and Pull" (mode 6), and "Pull and Push" (mode 7) used in [28]. For accommodating a wide range of desired accelerations for the piston of the pneumatic actuator in the positive direction, we would like to be able to appropriately mix modes 1 and 6. Similarly, for creating a wide range of desired piston accelerations in the negative direction, we need to appropriately mix modes 1 and 7. To this end, within each of the positive and negative actuation regions, we select a duty cycle based switching scheme that alternates between no actuation (mode 1) and full action (modes 6 and 7 in the positive and negative directions, respectively). Such a switching scheme is shown in Table II where

$$
d(u)=\frac{u_{H}-u}{u_{H}-u_{L}}
$$

with $u_{L} \leq u \leq u_{H}$. Note that in Table II, $u=1$ and $u=$ -1 correspond to the maximum actuation in the positive and negative directions, respectively. Also, $u=0$ corresponds to no actuation. Substituting (7) and the duty cycles $d_{1}, d_{6}$ and $d_{7}$ listed in Table II into (6), the average system model can be described by

$$
\dddot{y}_{a}= \begin{cases}f+b_{6} u & , \text { if } u \geq 0 \\ f+b_{7} u & , \text { if } u<0\end{cases}
$$




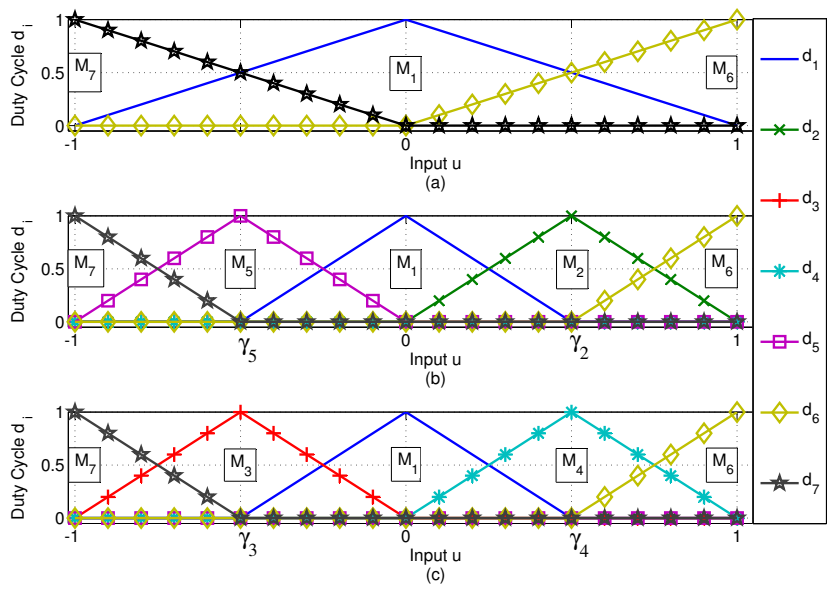

Fig. 2. Duty cycle mapping for: (a) the 3-mode system, (b) the 5-mode system or 7-mode system (pressurizing profile), or (c) the 7-mode system (venting profile).

TABLE II

THE 3-MODE DUTY CYCLE MAPPING PROFILE.

\begin{tabular}{cccc}
\hline \hline Region & $u_{L}$ & $u_{H}$ & Duty Cycles \\
\hline- & -1 & 0 & $d_{7}=d(u), d_{1}=1-d(u)$ \\
+ & 0 & 1 & $d_{1}=d(u), d_{6}=1-d(u)$ \\
\hline \hline
\end{tabular}

The scheme for switching between modes 1 and 6 or modes 1 and 7 according to the duty cycles listed in Table II is illustrated in Figure 2(a) as a function of the input $u$. Here, piecewise linear functions changing between 0 and 1 provide the duration for operating different mode. At any given input $u$ level, the duty cycle for high-frequency switching between the two modes present at that input level is determined by the durations $d_{i}$ 's for the two modes. Figure 3(a) shows (9) in the plane of $\dddot{y}_{a}-f$ versus $u$.

\section{B. Duty Cycle Mapping for the 5-Mode System}

If we extend the accepted values for the index $i$ in (7) to be $i \in\{1,2,5,6,7\}$, the system will include two more control options: "Push and Close" (mode 2) and "Close and Push" (mode 5). This mapping will result in a 5-mode system.

For a 5-mode system, similar to the 3-mode system, we need to decide the scheme for switching between modes. This mode selection scheme involves a new mapping of the single input $u$ to the duty cycle vector $D$. A desirable mapping would utilize at most two modes in any PWM period to simplify the mapping and also minimize the valves' switching for reduced noise and extended lifespan of the valves. Based on (7) and because $b_{i}$ in (3) are all positive, we can see that the openloop modes can be ordered in terms of the magnitude of the resulting $\dddot{y}$ for each mode as

$$
f_{7} \leq f_{5} \leq f_{1} \leq f_{2} \leq f_{6}
$$

Given this order of actuation level for each mode, it is beneficial to arrange the duty cycles as shown in Table III. Utilizing these mappings, the output $\dddot{y}_{a}$ is increasing (decreasing) with increasing (decreasing) $u$, only two modes are used at a time, and $\|D\|_{1}=1$. The mapping from Table III is plotted in
Figure 2(b). Figure 2(b) has four regions matching the same regions as in Table III.

TABLE III

THE 5-MODE DUTY CYCLE MAPPING OR THE 7-MODE PRESSURIZING PROFILE.

\begin{tabular}{cccc}
\hline \hline Region & $u_{L}$ & $u_{H}$ & Duty Cycles \\
\hline 1 & -1 & $-\gamma_{5}$ & $d_{7}=d(u), d_{5}=1-d(u)$ \\
2 & $-\gamma_{5}$ & 0 & $d_{5}=d(u), d_{1}=1-d(u)$ \\
3 & 0 & $\gamma_{2}$ & $d_{1}=d(u), d_{2}=1-d(u)$ \\
4 & $\gamma_{2}$ & 1 & $d_{2}=d(u), d_{6}=1-d(u)$ \\
\hline \hline
\end{tabular}

To properly select the values of the transition points $\gamma_{2}$ and $\gamma_{5}$ in Table III, we need to consider Figure 3(b). At $u=0$, mode 1 is utilized $100 \%$ of the time. As $u$ decreases into the negative values (region 2 in Table III), mode 5 is utilized increasingly until $u=-\gamma_{5}$, at which point mode 5 is utilized $100 \%$ of the time. As $u$ further decreases (region 1), mode 5 is used increasingly less, and mode 7 is utilized increasingly until $u=-1$, at which point mode 7 is utilized $100 \%$. The same holds for the positive range of $u$ corresponding to regions 3 and 4 in Table III. Now, if we select the following values for the transition points $\gamma_{2}$ and $\gamma_{5}$

$$
\gamma_{2}=\frac{b_{2}}{b_{6}} \quad \gamma_{5}=\frac{b_{5}}{b_{7}}
$$

then we can see from Figure 3(b) that the resulting averaged dynamics will demonstrate a straight line across the transition points. It should be noted that any other selection of $\gamma_{2}$ and $\gamma_{5}$ will lead to a non-uniformity in terms of actuation, which will not be beneficial to the controller design. Note that since $b_{i}$ in (3) are time-varying functions, $\gamma_{2}$ and $\gamma_{5}$ will also be functions of time.

Due to page limitation, the derivation of the time-averaged 5 -mode model is not shown in this paper. This derivation would show that, for the 5-mode system, the time-averaged model is the same as that for the 3-mode system. This is distinctly advantageous because we will be able to use the same control signal $u$ for both 3-mode and 5-mode systems. In this case, the only difference between 3-mode and 5-mode operation will be in the resulting valve open/close activity ${ }^{1}$ for a given $u$.

\section{Duty Cycle Mapping for the 7-Mode System}

Extending the accepted values for the index $i$ in (7) to $i \in$ $\{1, \ldots, 7\}$, the system will include two more control options: "Pull and Close" (mode 4) and "Close and Pull" (mode 3). This mapping will result in a 7-mode system.

For a 7-mode system, similar to the 5-mode system, a desirable mapping would utilize at most two modes in any PWM period. To properly evaluate the averaged model of the system, we will consider the 7-mode mapping through two separate mappings: the pressurizing profile (which utilizes modes $M_{7}, M_{5}, M_{1}, M_{2}$, and $M_{6}$ ) and the venting profile

\footnotetext{
${ }^{1}$ In this paper, an on/off valve's state going from 1 to 0 or from 0 to 1 is defined as one switch. The way we have measured the switching activity is the total count of switches made by all 4 solenoid valves divided by the total time.
} 
(which utilizes modes $M_{7}, M_{3}, M_{1}, M_{4}$, and $M_{6}$ ). Note that the mode selection scheme in the pressurizing profile in the 7 -mode case is the same as that in the 5-mode case. Based on (7) and because $b_{i}$ in (3) are all positive, we can see that these modes can be ordered in terms of the magnitude of the resulting $\dddot{y}$ for each mode as

$$
\begin{aligned}
\text { Pressurizing Profile: } & f_{7} \leq f_{5} \leq f_{1} \leq f_{2} \leq f_{6} \\
\text { Venting Profile: } & f_{7} \leq f_{3} \leq f_{1} \leq f_{4} \leq f_{6}
\end{aligned}
$$

Given this order of actuation for each mode, it is beneficial to arrange the duty cycles as shown in Table III for the pressurizing profile and Table IV for the venting profile. Observe that for the pressurizing profile of the 7-mode system, we are utilizing the same modes and mapping as the 5-mode system in the previous section.

TABLE IV

THE 7-MODE DUTY CYCLE MAPPING VENTING PROFILE.

\begin{tabular}{cccc}
\hline \hline Region & $u_{L}$ & $u_{H}$ & Duty Cycles \\
\hline 5 & -1 & $-\gamma_{3}$ & $d_{7}=d(u), d_{3}=1-d(u)$ \\
6 & $-\gamma_{3}$ & 0 & $d_{3}=d(u), d_{1}=1-d(u)$ \\
7 & 0 & $\gamma_{4}$ & $d_{1}=d(u), d_{4}=1-d(u)$ \\
8 & $\gamma_{4}$ & 1 & $d_{4}=d(u), d_{6}=1-d(u)$ \\
\hline \hline
\end{tabular}

Utilizing these mappings, the output $\dddot{y}_{a}$ is increasing (decreasing) with increasing (decreasing) $u$, only two modes are used at a time, and the $\|D\|_{1}=1$. The mapping in Tables III and IV are plotted in Figure 2(b) and 2(c).

We select the same values for the transition points $\gamma_{2}$ and $\gamma_{5}$ as in 5-mode (see (11)). Applying the same methodology to the venting profile, we select the following values for the transition points $\gamma_{4}$ and $\gamma_{3}$ in the venting profile:

$$
\gamma_{4}=\frac{b_{4}}{b_{6}} \quad \gamma_{3}=\frac{b_{3}}{b_{7}}
$$

Due to page limitation, the derivation of the time-averaged 7 -mode model is not shown in this paper. Interestingly, it can be shown that the time-averaged model of the 7-mode system is the same as that for the 3 -mode and 5-mode systems. So, we can use the same control input $u$ for 3-mode, 5-mode, and 7-mode systems.

\section{Sliding-Mode Control of a Single Pneumatic ACTUATOR}

In this section, we will be discussing the position control of a single actuator. For this purpose, we will need to develop a position controller for our pneumatic actuator. In the following, we will introduce a sliding scheme for position control.

Having expressed the multi-mode discrete-input system in the continuous-input form (9), a sliding-mode approach can be applied for position control of the system. Selecting an integral sliding surface as

$$
s_{p}=\left(\frac{d}{d t}+\omega_{p}\right)^{3} \int_{0}^{t} e_{p} d \tau
$$

where $e_{p}=y-y_{d}$ is the position error and $\omega_{p}$ is a positive gain. The switching function $s_{p}$ provides a measure of the distance from the sliding surface using the current position
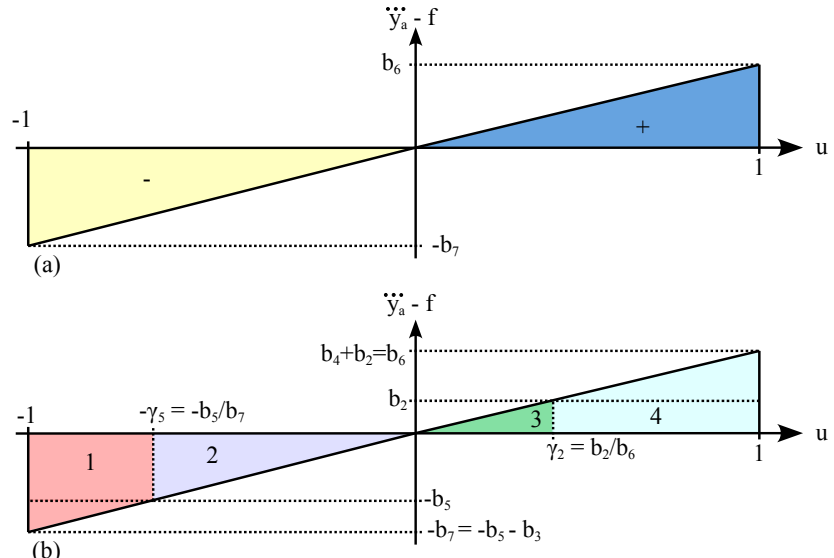

(b)

Fig. 3. Time-averaged input-output relationship for: (a) the 3-mode system, (b) the 5-mode system or 7-mode system (pressurizing profile).

error and its derivatives. One can develop a control law based on a sliding-mode approach, in which the equivalent control action, $u_{e q}$, is derived by solving for the input when $\dot{s}_{p}=0$. Taking the derivative of (11) we find

$$
\dot{s}_{p}=\dddot{y}-\dddot{y}_{d}+3 \ddot{e}_{p} \omega_{p}+3 \dot{e}_{p} \omega_{p}^{2}+e_{p} \omega_{p}^{3}
$$

If we substitute (9) as $\dddot{y}$ in (12), we obtain

$$
\dot{s}_{p}=f+\left(b^{+/-}\right) u-\dddot{y}_{d}+3 \ddot{e}_{p} \omega_{p}+3 \dot{e}_{p} \omega_{p}^{2}+e_{p} \omega_{p}^{3}
$$

where

$$
b^{+/-}=\left\{\begin{array}{l}
b_{6}, \text { if } u \geq 0 \\
b_{7}, \text { if } u<0
\end{array}\right.
$$

Solving for $u$ such that $\dot{s}_{p}=0$, we find $u_{e q}$ as

$$
u_{e q}=\frac{\hat{u}}{b^{+/-}}
$$

where

$$
\hat{u}=\dddot{y}_{d}-f-3 \omega_{p} \ddot{e}_{p}-3 \omega_{p}^{2} \dot{e}_{p}-\omega_{p}^{3} e_{p}
$$

Utilizing the control (14) alone does not ensure convergence to the sliding surface in finite time. Also, $\dddot{y}$ of the discreteinput system (actual system) and $\dddot{y}_{a}$ of the continuous-input system (average system) will be somewhat different. To study the robustness of the controller, let us model the actual $\dddot{y}$ as $\dddot{y}_{a}$ (from (9)) plus perturbations. We propose the following theorem where we augment the control action by a robustness term that also ensures convergence to the sliding surface in finite time.

\section{Theorem 1. Consider the perturbed system}

$$
\dddot{y}=\left(1+\Delta_{f}\right) f+\left(1+\Delta_{b}\right)\left(b^{+/-}\right) u
$$

where $\left|\Delta_{f}\right| \leq \alpha$ and $\left(\beta_{g m}\right)^{-1} \leq\left(1+\Delta_{b}\right) \leq \beta_{g m}$ (with $\left.\beta_{g m} \geq 1\right)$. The control input

$$
u=\frac{\hat{u}-K \operatorname{sgn}\left(s_{p}\right)}{b^{+/-}}
$$

with the time-variant robustness gain $K$

$$
K=\left(\beta_{g m}\left(\alpha|f|+\eta_{p}\right)+\left(\beta_{g m}-1\right)|\hat{u}|\right.
$$


will ensure convergence to the sliding surface $s_{p}=0$ in finite time where $s_{p}$ is defined in (11).

Proof. To be able to analyze the closed-loop stability, consider the Lyapunov function candidate

$$
V=\frac{1}{2} s_{p}^{2}>0
$$

If $\dot{V}<0$, then $V$ will be decreasing. If $V$ is decreasing, $\left|s_{p}\right|$ will also be decreasing. Assuming $s_{p}$ is initially bounded and $\left|s_{p}\right|$ is decreasing, then $s_{p}$ will be bounded and will asymptotically approach zero. Thus, we intend to control the system so that

$$
\dot{V}=\dot{s}_{p} s_{p} \leq-\eta_{p}\left|s_{p}\right|
$$

Substituting (15), (16), and (17) into (12), we find

$$
\dot{s}_{p}=\left(b^{+/-}\right) u-\hat{u}+\left(\Delta_{f}\right) f+\left(\Delta_{b}\right)\left(b^{+/-}\right) u
$$

Using (17) and (21), we find

$$
\dot{s}_{p}=-K \operatorname{sgn}\left(s_{p}\right)+\left(\Delta_{f}\right) f+\left(\Delta_{b}\right)\left(b^{+/-}\right) u
$$

Substituting (18) into (22), we find

$$
\begin{aligned}
\dot{s}_{p} & =-\operatorname{sgn}\left(s_{p}\right)\left[\beta_{g m}\left(\alpha|f|+\eta_{p}\right)-\operatorname{sgn}\left(s_{p}\right)\left(\Delta_{f}\right) f\right. \\
& \left.+\left(\beta_{g m}-1\right)|\hat{u}|-\operatorname{sgn}\left(s_{p}\right)\left(\Delta_{b}\right)\left(b^{+/-}\right) u\right]
\end{aligned}
$$

Given that $\left(\beta_{g m}-1\right) \geq\left(\Delta_{b}\right)$, and $|\hat{u}| \geq \operatorname{sgn}\left(s_{p}\right)\left(b^{+/-}\right) u$, we find that

$$
\left(\beta_{g m}-1\right)|\hat{u}|-\operatorname{sgn}\left(s_{p}\right)\left(\Delta_{b}\right)\left(b^{+/-}\right) u \geq 0
$$

and thus (23) can be simplified to

$$
\dot{s}_{p} \leq-\operatorname{sgn}\left(s_{p}\right)\left[\beta_{g m}\left(\alpha|f|+\eta_{p}\right)-\operatorname{sgn}\left(s_{p}\right)\left(\Delta_{f}\right) f\right]
$$

Also, given that $\beta_{g m} \geq 1$, and $\alpha \geq \Delta_{f}$, we find

$$
\beta_{g m} \alpha|f|-\operatorname{sgn}\left(s_{p}\right)\left(\Delta_{f}\right) f \geq 0
$$

and thus (25) can be simplified to

$$
\dot{s}_{p} \leq-\beta_{g m} \eta_{p} \operatorname{sgn}\left(s_{p}\right)
$$

Since $\beta_{g m} \geq 1$, we get

$$
\dot{s}_{p} \leq-\eta_{p} \operatorname{sgn}\left(s_{p}\right)
$$

Multiply both sides of (28) by $s_{p}$ and we obtain

$$
\dot{s}_{p} s_{p} \leq-\eta_{p}\left|s_{p}\right|
$$

Thus, the system will converge to the sliding surface $s_{p}=0$ in finite time because of (29).

The above controller leads to the closed-loop stable dynamics

$$
\left(\frac{d}{d t}+\omega_{p}\right)^{3} \int_{0}^{t} e_{p} d \tau=0
$$

in which the position error $e_{p}$ asymptotically tends towards zero. Utilizing the control action $u$ obtained from (2), (15), (17), and (18), we can apply the closed-loop control to a 3mode system using the mapping from Table II or to a 7-mode system using the mapping from Tables III and IV. For the 7mode mapping there are two separate profiles: the venting and pressurizing profiles. The selection between the pressurizing and venting profiles is updated periodically based on the larger output actuation $b_{i}$. The order of the two modes in any given PWM window was arranged to minimize the overall switching activity. For example, if a PWM window ended with mode $M_{1}$ and the next window contained the $M_{1}$ mode, that mode was used at the start of the next window.

\section{EXPERIMENTAL RESULTS}

\section{A. Experimental Setup}

In this paper, experiments were performed with a pair of 1-DOF pneumatic actuators as the master and the slave (see Figure 4). The low friction cylinders (Airpel model M16D100D) have a $16 \mathrm{~mm}$ diameter and a $100 \mathrm{~mm}$ stroke. The piston and shaft mass is approximately $\mathrm{M}=900 \mathrm{~g}$. The pneumatic solenoid valves (Matrix model GNK821213C3K) used to control the air flow have switching times of approximately $1.3 \mathrm{~ms}$ (opening time) and $0.2 \mathrm{~ms}$ (closing time). With such fast switching times, the on/off valves are appropriate for the purposes of the proposed control. In terms of sensors, a low-friction linear variable differential transformer (LVDT) is connected to the cylinder in order to measure the linear positions. The force sensor used is an ELPF Load Cell model, manufactured by Measurement Specialties, Inc. The controller is implemented using a dSPACE board (DS1104), running at a sampling rate of $500 \mathrm{~Hz}$. This sampling rate $500 \mathrm{~Hz}$ has been chosen according to the open/close bandwidth of the valves opening and closing time smaller than $1.3 \mathrm{~ms}$ and to enable an acceptable tracking response. The PWM period has been set to $10 \mathrm{~ms}$. We could not use the built-in dSPACE PWM outputs to generate the PWM signals as we needed to synchronize and precisely control the 4 independent signals (each pair of duty cycles changed every period). So, we built our own PWM signal generation through software. Knowing that the sampling period is $2 \mathrm{~ms}$ and the PWM period $10 \mathrm{~ms}$, we obtained a PWM generation with a resolution of 0.2 . The controller switching function employs the first and second derivatives of the force error. This latter is obtained by a numerical differentiation of the force twice. Since the signal processing produces a considerable amount of numerical noise. We compensate for this by filtering the measured force error by a 2nd order lowpass Butterworth filter with a cutoff of $10 \mathrm{~Hz}$. This reduces this noise to an acceptable level but introduces a small delay which the sliding-mode control successfully compensates for. Note that the values of $b^{+/-}$are computed in real time at each sampling time. The experimental setup has the model parameters listed in Table V.

TABLE V

SYSTEM'S MODEL PARAMETERS.

\begin{tabular}{ccl}
\hline \hline Var. & Value & Label \\
\hline$l$ & $0.1 \mathrm{~m}$ & Chamber Length \\
$T$ & $296 \mathrm{~K}$ & Supply Temperature \\
$P_{S}$ & $300,000 \mathrm{~Pa}$ & Supply Air Pressure \\
$P_{E}$ & $100,000 \mathrm{~Pa}$ & Exhaust Air Pressure \\
$k$ & 1.2 & Polytropic Constant \\
$A_{P}, A_{N}$ & $1.814 \mathrm{~cm}^{2}$ & Piston Cylinder Area \\
$b_{m}$ & $50 \mathrm{~N} \mathrm{~s} / \mathrm{m}$ & Viscosity Coefficient \\
$M$ & $0.9 \mathrm{~kg}$ & Total Mass of load \\
\hline \hline
\end{tabular}




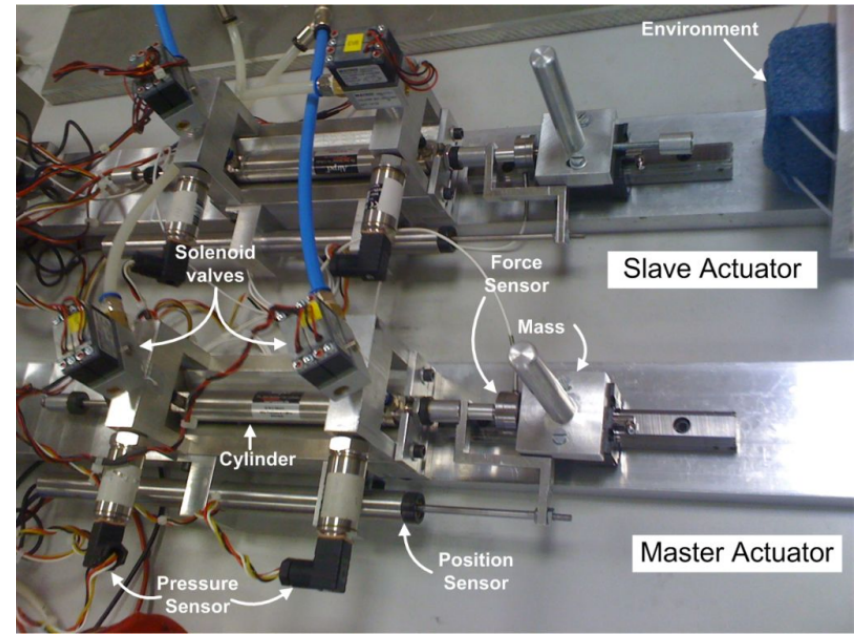

Fig. 4. Experimental setup.

\section{B. 7-Mode Position Control of a Single Actuator}

This section shows the experimental results conducted with the 3-mode and 7-mode model based controllers (MBC3 and MBC7) proposed in this paper. These results are compared to the ones obtained for the controllers introduced in [30] (Switched Controller with 3 and 7 modes: SC3 and SC7). The parameters utilized for the MBC Controllers (based on equations (15), (17), and (18) ) are $\omega_{p}=60 \mathrm{rad} / \mathrm{s}, \alpha=0.1$, $\beta_{g m}=1.1$, and $\eta_{p}=100 \mathrm{~m} / \mathrm{s}^{3}$.

1) Position tracking performances: in order to highlight the enhancements of the MBC controllers in comparison with the SC ones, we performed a series of $4 \mathrm{~cm}$ step responses (around the middle of the piston range). Figure 5 gathers the results obtained with the MBC and SC controllers, for 3 and 7 modes. These plots display the desired and real trajectories, the tracking errors and the switches for solenoid valves 1 and 2 : positive (resp. negative) values indicate that the chamber $\mathrm{P}$ is connected to supply (resp. exhaust). This permits to get an overview of the switching activity. These plots show very close performances from a qualitative point of view. The MBC controllers appear to provide slightly better performances.

Table VI shows the RMS value of the tracking error $\left(\varepsilon_{R M S}\right)$ provided in $\mathrm{mm}$ and also in percentage of the step amplitude, and the Total Number of Switches per Second (TNSS) of the four solenoid valves. The RMS values of the tracking error for each controller are very close. They slightly differ from an experiment to another because the dry friction induced by the mechanical guiding rail is not repetitive. The TNSS appears to be the lower for MBC than SC and it decreases from 3-modes to 7 -modes.

The conclusion we can draw is that tracking performances are close between both control laws but we obtain much better performances in terms of switching and so in term of solenoid valve lifespan with the MBC controllers.

2) Precision performances: in order to analyze the performances of each controller in terms of precision, we performed a series of experiments where the reference signal is made of
TABLE VI

RMS AND TOTAL NUMBER OF SWITCHES FOR SC AND MBC CONTROL LAWS IN 3 AND 7 MODES

\begin{tabular}{lccr}
\hline \hline Controller & $\varepsilon_{R M S}(\mathrm{~mm})$ & $\varepsilon_{R M S}(\%)$ & TNSS (Hz) \\
\hline SC3 & 6.3 & 1.6 & 149 \\
SC7 & 6.2 & 1.5 & 70 \\
MBC3 & 6.4 & 1.6 & 46 \\
MBC7 & 6.4 & 1.6 & 38 \\
\hline \hline
\end{tabular}
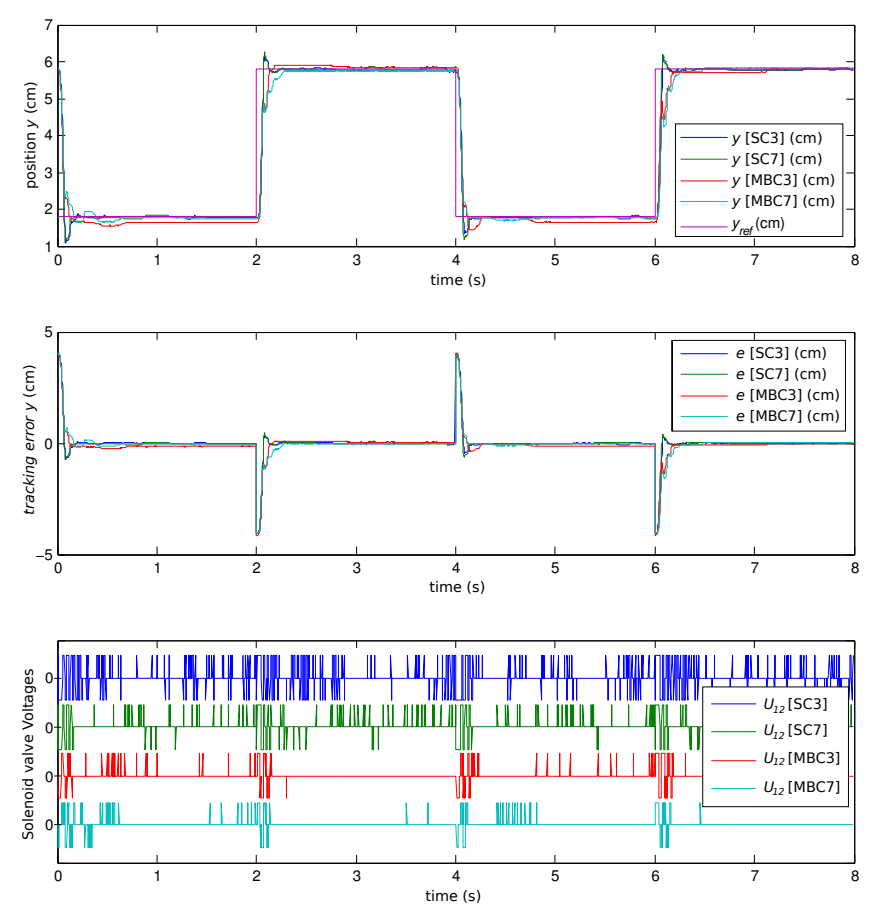

Fig. 5. Performance comparison for step responses with control laws from [30] and a moving mass of $900 \mathrm{~g}$.

50 successive steps from a central position $(3.5 \mathrm{~cm})$ with an amplitude of $1.5 \mathrm{~cm}$. For the visualization of the results, only the first 10 seconds of these steps are depicted in figure 6.

Table VII gathers the different quantitative results obtained with the four controllers, namely, the RMS value of the tracking error $\varepsilon_{R M S}$, its average value $|\bar{\varepsilon}|$, the amplitude of its oscillations $|\Delta \varepsilon|$ and the TNSS during steady state periods. Table VIII provides quantitative results for during the transient responses: average overshoot amplitude $\overline{\left|y_{\text {over }}\right|}$ and the TNSS during these periods.

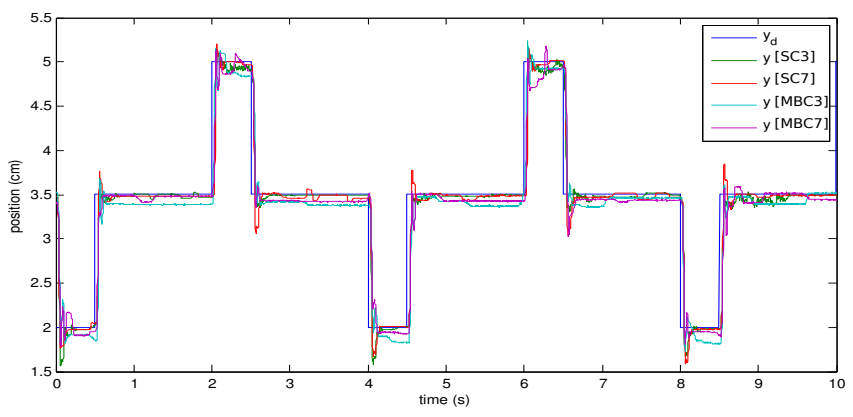

Fig. 6. A few $\pm 1.5 \mathrm{~cm}$ step responses around a central position. 
TABLE VII

STEADY STATE ANALYSIS FOR 50 STEP RESPONSES WITH THE FOUR CONTROLLERS.

\begin{tabular}{lccccr}
\hline \hline Control law & $\varepsilon_{R M S}(\mathrm{~mm})$ & $\varepsilon_{R M S}(\%)$ & $|\bar{\varepsilon}|(\mathrm{mm})$ & $|\Delta \varepsilon|(\mathrm{mm})$ & TNSS (Hz) \\
\hline SC3 & 0.4 & 2.4 & 0.3 & 2.5 & 148 \\
SC7 & 0.3 & 2.0 & 0.2 & 1.7 & 57 \\
MBC3 & 0.8 & 5.4 & 0.5 & 5.1 & 43 \\
MBC7 & 0.7 & 4.4 & 0.5 & 5.3 & 31 \\
\hline \hline
\end{tabular}

TABLE VIII

TRANSIENT RESPONSES FOR 50 STEP RESPONSES WITH THE FOUR CONTROLLERS.

\begin{tabular}{lccc}
\hline \hline Controller & $\overline{\left|y_{\text {over }}\right|}(\mathrm{mm})$ & $\overline{\left|y_{\text {over }}\right|}(\%)$ & TNSS (Hz) \\
\hline SC3 & 1.1 & 7,4 & 254 \\
SC7 & 2.7 & 18 & 188 \\
MBC3 & 1.4 & 9,6 & 229 \\
MBC7 & 2.3 & 15 & 189 \\
\hline \hline
\end{tabular}

These results show clearly that better results in term of absolute precision in steady state for the SC controller whereas the TNSS is better for the MBC one. During the transient response, the mean overshoots for $\mathrm{SC}$ and $\mathrm{MBC}$ are very close but the standard deviation is higher which explains that the maximum overshoot for MBC7 is much higher than others. Furthermore, since the system moves fastly during the transient response, the TNSS shows close results in terms of switching for SC and MBC controllers. We can draw the same conclusions as the previous ones with these measures.
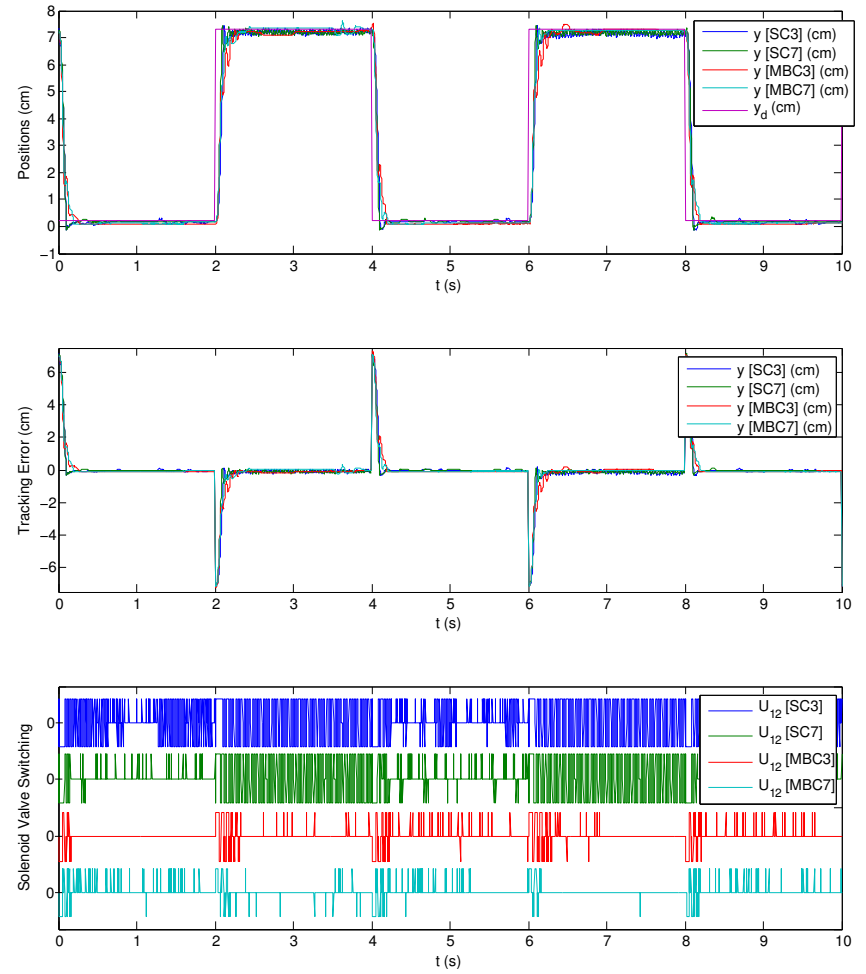

Fig. 7. Step responses for large amplitudes for SC and MBC controllers.

3) Large steps: we also performed experiments with the largest displacements we can afford on the test bed in order to check the behavior of the system when the load is near to the end of the stroke. Results could be seen in Figure 7.
TABLE IX

RMS AND TNSS FOR LARGE STEPS FOR SC AND MBC CONTROLLERS.

\begin{tabular}{lccr}
\hline \hline Controller & $\varepsilon_{R M S}(\mathrm{~mm})$ & $\varepsilon_{R M S}(\%)$ & TNSS (Hz) \\
\hline SC3 & 12.6 & 17.7 & 259 \\
SC7 & 11.9 & 16.8 & 92 \\
MBC3 & 12.0 & 16.9 & 42 \\
MBC7 & 11.8 & 16.6 & 25 \\
\hline
\end{tabular}

We can observe that at the end of the stroke, the MBC controller does not deteriorate the position tracking (while the SC3 controller provokes visible vibrations) but it is at the cost of more switches (performance numbers are listed in table IX.)

4) Robustness with respect to moving mass variations: in order to test the robustness of our algorithm when the model mass differs from the real moving one, we performed a series of experiments with a model mass of $1.4 \mathrm{~kg}$ and three different moving masses: $0.9 \mathrm{~kg}, 1.4 \mathrm{~kg}$ and $1.9 \mathrm{~kg}$. Figure 8 shows the global response of the system to a same sawtooth reference signal. Table $\mathrm{X}$ details the numerical results in terms of error and number of switches: RMS (absolute and relative) errors in 3 and 7 modes for three different real masses when the model mass is $1.4 \mathrm{~kg}$.
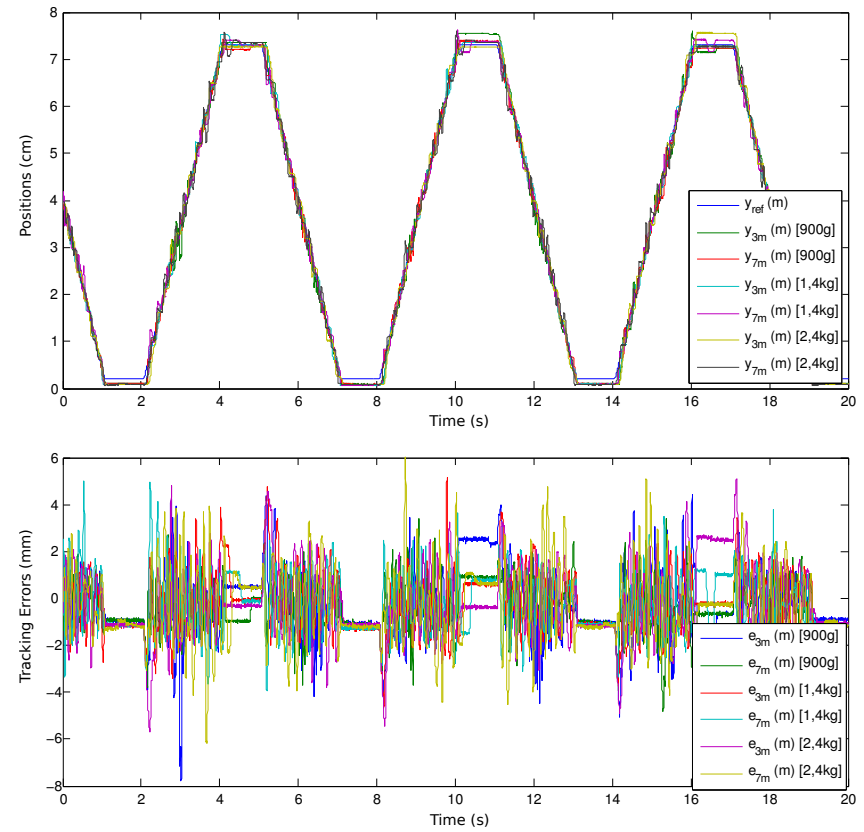

Fig. 8. Sawtooth experiments for a model mass of $1.4 \mathrm{~kg}$ and different real moving masses $(0.9 \mathrm{~kg}, 1.4 \mathrm{~kg}$ and $1.9 \mathrm{~kg})$ with $\mathrm{MBC}$ controller.

It appears from the plots and the numerical results that the performances of the position tracking are the same for $\pm 35 \%$ of difference between the model and the actual mass. The additional mass create nonlinear friction which make the experiments not repeatable but the results remain close.

5) Harmonic response: We also studied the performances of this system through harmonic tests. The following sine wave test pattern was used to test the position tracking performance of the proposed algorithm:

$$
y_{d}=20 \mathrm{~mm} \sin (2 \pi f t)
$$


TABLE X

TRACKING ERRORS IN 3 AND 7 MODES FOR THREE DIFFERENT REAL MASSES WHEN THE MODEL MASS IS 1.4KG (MBC CONTROLLER).

\begin{tabular}{lccc}
\hline \hline Control law & Real mass $(\mathrm{kg})$ & Absolute RMS Error $(\mathrm{mm})$ & Relative RMS Error $(\%)$ \\
\hline 3 modes & 0.9 & 1,41 & 1,99 \\
7 modes & 0.9 & 1,06 & 1,49 \\
\hline 3 modes & 1.4 & 1,27 & 1,76 \\
7 modes & 1.4 & 1,28 & 1,80 \\
\hline 3 modes & 1.9 & 1,57 & 2,22 \\
7 modes & 1.9 & 1,53 & 2,16 \\
\hline \hline
\end{tabular}
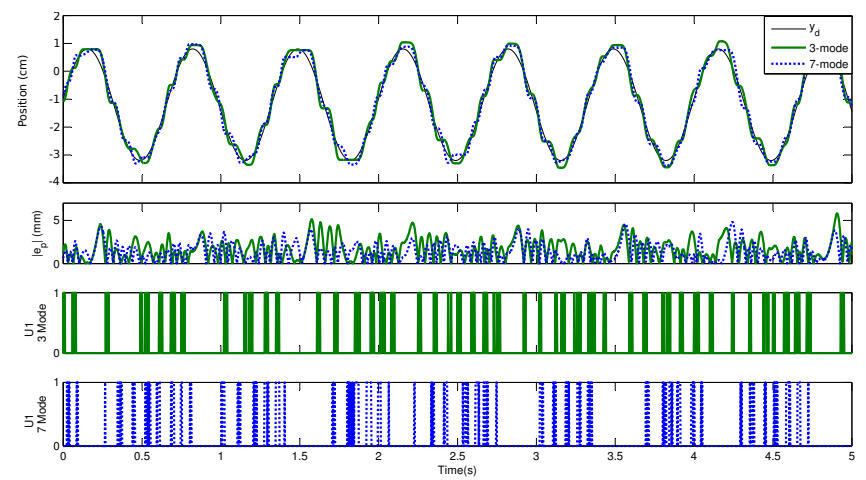

Fig. 9. Tracking and switching performance of a single actuator with a $1.5 \mathrm{~Hz}$ sine wave input under no load on pulley (MBC controller).

The frequency was varied from $0.1 \mathrm{~Hz}$ to $3.0 \mathrm{~Hz}$. The tracking performance and switching activity was evaluated for this test pattern over a 10 second period. Please refer to Figure 9 for an example at $1.5 \mathrm{~Hz}$.

The results for different sine wave frequencies are plotted in Figure 10. From these results, we find that for both the 3mode and the 7-mode systems, increasing the input frequency increases the RMS tracking error. When we compare the results for the 3-mode controller and the 7-mode controller, we can see that for the latter there are notable decreases in both the tracking error and the switching activity. 5-mode control performances are not presented in this paper as they are located between 3 and 7 mode.

To test the system's ability to reject external force disturbance, the experiment was run again utilizing the sinewave test input with a weight attached to the actuator via a cord and pulley (see Figure 13). The weights tested were $0.5 \mathrm{~kg}$ (Figure 11) and $1.0 \mathrm{~kg}$ (Figure 12). These weights applied a constant gravity force in the positive direction of the actuator. To prevent the weights attached to the actuator from pendulum-like swinging, only frequencies from $0.1 \mathrm{~Hz}$ to $1.5 \mathrm{~Hz}$ were tested. As the results show, tracking error was not significantly increased as a result of attaching the weights, and valve switching activity was increased only marginally. This demonstrates the robustness of the controller to external force disturbances.

Even if we stay in a half step mode (modes 2-5), the solenoid valves may continue to switch each PWM period to maintain actuation. However these half step modes involve half as much switching. With smaller actuation we will get a lower total number of switches.
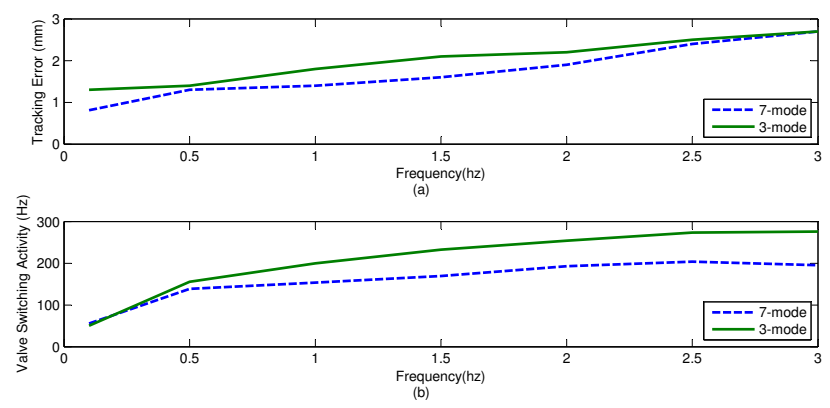

Fig. 10. Tracking and switching harmonic performance of a single actuator with a sine wave input with no load on the pulley (MBC controller).
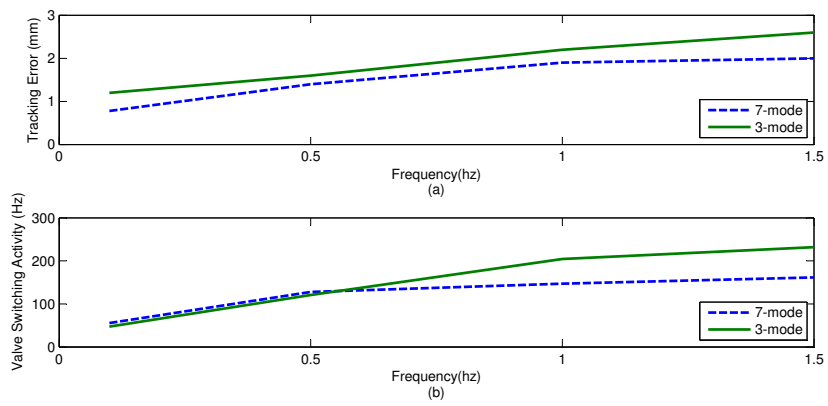

Fig. 11. Tracking and switching harmonic performance of a single actuator with a sine wave input with $0.5 \mathrm{~kg}$ mass on the pulley (MBC controller).
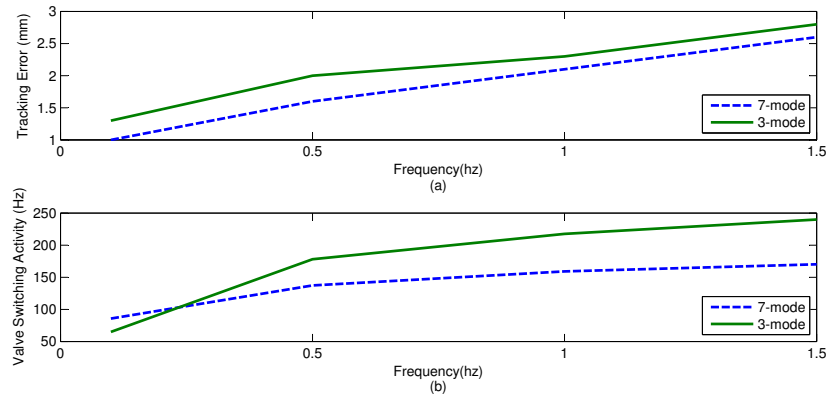

Fig. 12. Tracking and switching harmonic performance of a single actuator with a sine wave input with $1.0 \mathrm{~kg}$ mass on the pulley (MBC controller).

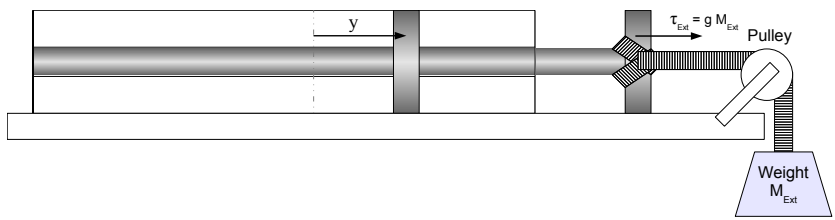

Fig. 13. Experimental external force disturbance loading. 
TABLE XI

MASTER/SLAVE ACtUATOR VARIABle NAMES WHERE $q \in\{P, N\}, v \in\{1-7\}$, AND $t \in\{1-7\}$

\begin{tabular}{ccccccc}
\hline \hline Single actuator & $y$ & $P_{q}$ & $V_{q}$ & $\tau_{E x t}$ & $b_{t}$ & $f$ \\
\hline Master & $y_{m}$ & $P_{q, m}$ & $V_{q, m}$ & $\tau_{h}$ & $b_{v, m}$ & $f_{m}$ \\
Slave & $y_{s}$ & $P_{q, s}$ & $V_{q, s}$ & $-\tau_{e}$ & $b_{t, s}$ & $f_{s}$ \\
\hline \hline
\end{tabular}

\section{Teleoperation Control of Two Actuators}

This section of the paper focuses on teleoperation control of a pair of pneumatic actuators, called "master" and "slave". The master and slave dynamics will be the same as those described in Section II, with the difference that the common variables will be re-labelled as shown in Table XI.

The block diagram in Figure 14 shows the architecture of a force-position bilateral teleoperation system. The slave side of the setup utilizes the sliding control of position described in Section IV, where $e_{p}$ is defined as $y_{m}-y_{s}$. The master side of the setup utilizes the switching function based control of force described in Appendix A, where $e_{f}$ is defined as $-\tau_{h}-\tau_{e}$ (net force difference despite the signs).

To test the teleoperation control scheme developed above, a quasi-periodic input motion pattern was applied by the human operator's hand to the master. This input resembled three cycles of back-and-forth motion with an approximately $10 \mathrm{~mm}$ RMS amplitude when the slave was in free space, followed by approximately two seconds of motion causing contact between the slave and its environment. The slave's environment was a soft material located $14.5 \mathrm{~mm}$ away from the slave's zero position. This entire motion pattern was repeated three times over a 20 second period by the human operator. The position and force profiles of the master and the slave actuators were measured via position and force sensors (see Figure 4).

For this experiment, the following parameters were selected for the master controller in (B1): $\omega_{f}=50 \mathrm{rad} / \mathrm{s}, \tau=40 \mathrm{~ms}$, $\epsilon=0.5 \mathrm{~N}, \beta=1.7 \mathrm{~N}$, and $\xi=1$. The slave controller utilized the same control parameters described in Section V-B.

The results are depicted in Figure 15. Since the slave actuator has a soft material located at $14.5 \mathrm{~mm}$ from it, when the operator tries to move the slave beyond that position, the master reflects a force to the operator that is proportional to the measured contact force between the slave and the environment. The force-position scheme relied either on the 3 -mode or the 7-mode based sliding control of the slave actuator's position. Comparing the results between the 3-mode and the 7-mode cases in Figure 15, we can see that there is an $11 \%$ improvement in the RMS error of position tracking error with the 7-mode based control compared to the 3-mode based control.

When the slave actuator's position is less then $14.5 \mathrm{~mm}$, the slave is in free motion (i.e., $\tau_{e} \approx 0$ ). Under the free motion condition, the position tracking and force tracking were not found to significantly improve under the 7-mode based control when compared to the 3-mode based control under force-position control. Nonetheless, going from the 3-mode to the 7 -mode controller, we see a consistent $20 \%$ reduction in switching activity of the on/off solenoid valves, which increases their operating lifespan. For the 3-mode controller,
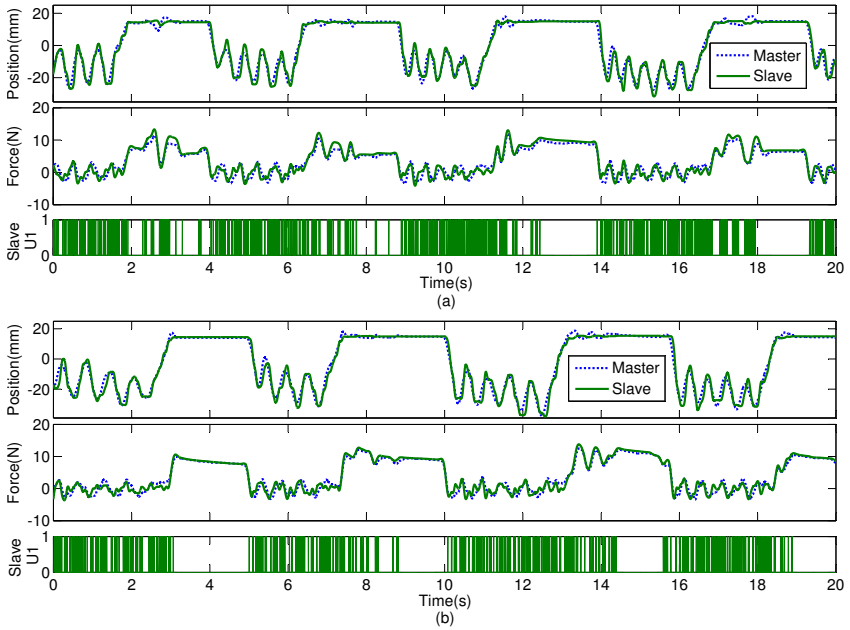

Fig. 15. Force-position control with Model Based Controller on the slave for: (a) 3-mode, and (b) 7-mode.

a total of 4792 switches were counted, while for the 7-mode controller, a total of 3782 switches were counted.

To explain this switching activity upgrade, we observe in figure $15 \mathrm{~b}$ that, in 3 mode, the controller switches between full actuation (modes 6/7) and no actuation (mode 1). Whereas in 7 mode, the controller switches depend on the region of actuation which includes half steps (modes 2-5). Since the actuation resolution is higher, the system remains longer in steady state mode. Mode M1 is activated (duty cycles are equal to $0 \%$ ) between $3 \mathrm{~s}$ and $5 \mathrm{~s}$.

\section{CONCLUding REMARKS}

In this paper, we created a 7-mode mapping for the Model Based Controller to obtain a continuous input time-averaged dynamic model. This time-averaged model was utilized to create a sliding control law for position control of a single pneumatic actuator. For this sliding control, we developed a stability proof demonstrating convergence of the sliding surface in finite time (and thus asymptotic convergence of the position error). This 7-mode architecture, was then expanded to a paired actuator setup utilizing a force-position teleoperation control scheme.

The 7-mode control was compared experimentally against the 3-mode control in terms of tracking performance and switching activity for both the single actuator and the teleoperated pair of actuators. For one actuator, the 7-mode controller showed the best compromise between position tracking performance and reduced valve switching activity. For the teleoperation system, in contact motion the 7-mode system was found to show a significant improvement in terms of position tracking, and switching activity over the 3-mode system. Thus, for both a single actuator and a teleoperation system, the performance was found to improve with the additional modes of actuation. These additional modes of operation provided by the 7-mode controller allowed for reduced, yet appropriate amounts of drive actuation. The outcome was more efficient actuator control. As the compliance of the cylinder is different between M1 and M8 or M9, Future works will study the 


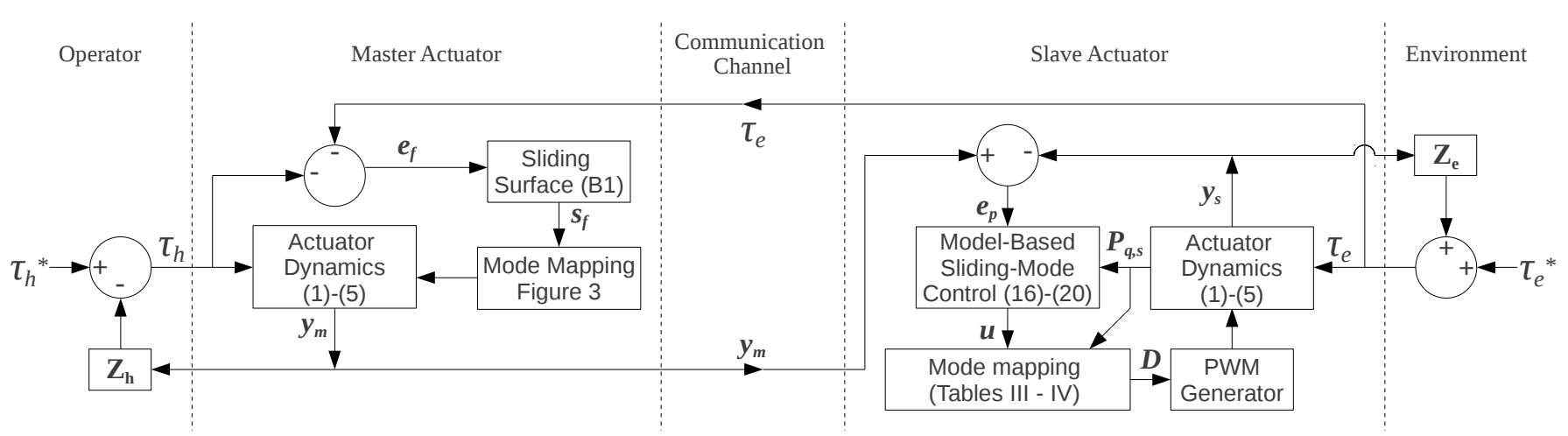

Fig. 14. Force-position teleoperation control.

performances of a 9 mode compliant control law to enhance the transparence of the teleoperation loop.

\section{APPENDIX}

\section{A. Time-Averaged Model for the 7-Mode System}

For a force-controlled system, let us define the sliding surface as $s_{f}=0$ where the switching function $s_{f}$ is

$$
s_{f}=\frac{\ddot{e}_{f}}{\omega_{f}^{2}}+\frac{2 \xi \dot{e}_{f}}{\omega_{f}}+e_{f}
$$

Here, $e_{f}=\tau-\tau_{d}$ is the force error. Also, $\xi$ and $\omega_{f}$ are constant and positive numbers. Thus, the switching function $s_{f}$ provides a measure of the distance from the sliding surface using the current force error and its derivatives. Take the derivative of (B1) to get

$$
\begin{aligned}
\dot{s}_{f} & =\frac{\dddot{e}_{f}}{\omega_{f}^{2}}+\frac{2 \xi \ddot{e}_{f}}{\omega_{f}}+\dot{e}_{f} \\
& =\frac{\dddot{e}_{f}}{\omega_{f}^{2}}+\frac{2 \xi \ddot{e}_{f}}{\omega_{f}}+\left(\dot{\tau}-\dot{\tau}_{d}\right)
\end{aligned}
$$

Given that $\dot{\tau}=M \dddot{y}$, we can find $\dot{s}_{f}$ by substituting (3) into (B2):

$$
\dot{s}_{f}= \begin{cases}\lambda & , \text { mode } M_{1} \\ \lambda+(-1)^{i} b_{i} M & , \text { mode } M_{i}, \quad(2 \leq i \leq 7)\end{cases}
$$

where $\lambda=M f+\dot{\tau}_{E x t}-\dot{\tau}_{d}+\dddot{e}_{f} / \omega_{f}^{2}+2 \xi \ddot{e}_{f} / \omega_{f}$.

To ensure convergence of the sliding surface $s_{f}=0$ we wish to control the system such that $\dot{s}_{f}$ is always approaching the sliding surface. For higher force tracking accuracy and less switching we utilize five different regions of the function $s_{f}$. These regions of $s_{f}$ and the selected operating mode of the system are illustrated in Table XII.

If we utilize a pneumatic controller based on Table XII, for the lowest error $\left|s_{f}\right|<\epsilon$, we use the mode $M_{1}$ which has no active effect on the system (i.e., no actuation). For the positive error $s_{f}>\beta$, we use the mode $M_{7}$, which exerts the highest $\dot{s}_{f}$ in the negative direction. Conversely, for the largest negative error $s_{f}<-\beta$, we utilize the mode $M_{6}$, which generates the highest $\dot{s}_{f}$ in the positive direction. Evidently, this controller ensures that the system is always approaching
TABLE XII

SELECTION OF THE OPERATING MODE BASED ON FORCE ERROR $s_{f}$.

\begin{tabular}{ccc}
\hline \hline Region of $s_{f}$ & $\begin{array}{c}\text { Selected } \\
\text { operating mode }\end{array}$ & $\begin{array}{c}\text { Resulting magnitude } \\
\text { of } \dot{s}_{f} \text { from (B3) }\end{array}$ \\
\hline$s_{f}>\beta$ & $M_{7}$ & Large negative \\
$\beta \geq s_{f}>\epsilon$ & $M_{3}$ and $M_{5}$ & Medium negative \\
$\epsilon \geq s_{f}>-\epsilon$ & $M_{1}$ & Minimal \\
$-\epsilon \geq s_{f}>-\beta$ & $M_{2}$ and $M_{4}$ & Medium positive \\
$-\beta \geq s_{f}$ & $M_{6}$ & Large positive \\
\hline \hline
\end{tabular}

the $s_{f}=0$ sliding surface (assuming the closed-loop system remains stable).

This control architecture presents unique control choices when $\left|s_{f}\right|>\beta$ or $\left|s_{f}\right|<\epsilon$ but not when $\beta \geq\left|s_{f}\right| \geq \epsilon$. For the positive and the negative regions, there are the two mode combinations $\left(M_{3}\right.$ and $\left.M_{5}\right)$ and $\left(M_{2}\right.$ and $\left.M_{4}\right)$, respectively. In fact, each region requires a choice between whether it is better to vent one chamber or pressurize the other one. In the following, we propose a scheme to make this choice.

Assuming we have pressure sensors in each chamber of the actuator, we can use this additional state measurement to choose the mode that will generate a larger magnitude of $\dot{s}_{f}$ (i.e., a more forceful control action). To this end, let us define a nominal quantity $E_{1}$ as

$$
E_{1}=\left(P_{S}+P_{E}\right)-\left(P_{P}+P_{N}\right)
$$

If $E_{1}$ is positive, then the global pressure (or sum of pressures) in the two chambers $\left(P_{P}\right.$ and $\left.P_{N}\right)$ is lower than the global input pressures $\left(P_{S}\right.$ and $\left.P_{E}\right)$. In this case, we may conclude that pressurizing will lead to a larger magnitude of $\dot{s}_{f}$ than venting.

The above conclusion is true because the magnitude of output force is proportional to the difference in pressure between the two chambers $P_{P}$ and $P_{N}$. If the time to fully vent or pressurize a chamber is small, then we can conclude that the piston acceleration will be larger when pressurizing when the global chamber pressure is lower than the nominal value.

Conversely, if $E_{1}$ is negative, then the global pressure in the two chambers $\left(P_{P}\right.$ and $\left.P_{N}\right)$ is higher than the global input pressures $\left(P_{S}\right.$ and $\left.P_{E}\right)$. As a result, venting will have a larger magnitude of $\dot{s}_{f}$ then pressurizing. 


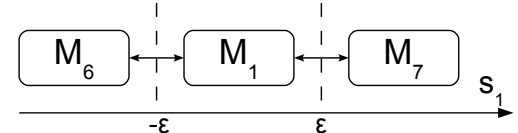

(a)

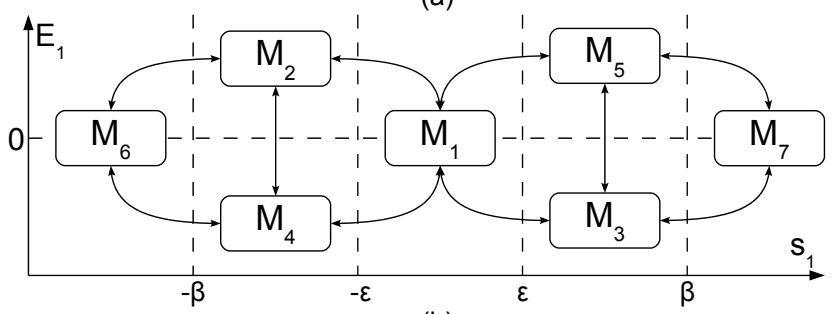

(b)

Fig. 16. Mode mapping for sliding-mode control force control for: (a) the 3-mode system, (b) the 7-mode system.

To reduce switching activity, the change from one chamber pressurizing to the other chamber venting was limited to occurring once every $\tau$ seconds. This implementation of a pneumatic 7-mode controller is illustrated in Figure 16(b). Note that in the 3-mode and 5-mode cases, this analysis was not necessary. The pneumatic 3-mode controller is illustrated in Figure 16(a).

\section{ACKNOWLEDGMENT}

This research was supported by the Natural Sciences and Engineering Research Council (NSERC) of Canada and the Centre National pour la Recherche Scientifique (CNRS) in France. The authors would like to thank Christophe Ducat for the mechanical design of the test bed.

\section{REFERENCES}

[1] J. M. Sabater, M. Hernandez, R. J. Saltarén, R. Aracil, E. Yime, and J. M. Azorín, "Teleoperated parallel climbing robots in nuclear installations," Industrial Robot: An International Journal, vol. 33, no. 5, pp. 381-386, September 2006.

[2] M. Q. Le, M. T. Pham, M. Tavakoli, and R. Moreau, "Sliding mode control of a pneumatic haptic teleoperation system with on/off solenoid valves," in Proceedings of 2011 IEEE International Conference on Robotics and Automation (ICRA), Shanghai, China, May 2011, pp. 874 879.

[3] G. S. Fischer, I. Iordachita, C. Csoma, J. Tokuda, S. P. DiMaio, C. M. Tempany, N. Hata, and G. Fichtinger, "MRI-compatible pneumatic robot for transperineal prostate needle placement," IEEE/ASME Transactions on Mechatronics, vol. 13, no. 3, pp. 295-305, 2008.

[4] N. Sepehri and P. D. Lawrence, "Model-based sensor-based velocity control of teleoperated heavy-duty hydraulic machines," in Proceedings of the IEEE/RSJ International Conference on Intelligent Robots and Systems, Raleigh, NC, July 1992, pp. 859-864.

[5] S. Lee, M. Kim, and C.-W. Lee, "Human-robot integrated teleoperation," Advanced Robotics, vol. 13, no. 4, pp. 437-439, 1999.

[6] M. Girone, G. Burdea, M. Bouzit, V. Popescu, and J. Deutsch, "Stewart platform-based system for ankle telerehabilitation," Autonomous Robots, vol. 10, no. 2, pp. 203-212, 2001.

[7] J. A. Rosas-Flores, J. A. Flores-Campos, and L. G. Corona-Ramírez, "Optimal linearization of the dynamic behavior of an on/off actuated single pneumatic cylinder," in Proceedings of 2008 5th International Conference on Electrical Engineering, Computing Science and Automatic Control (CCE 2008), Mexico City, November 2008, pp. 380-385.

[8] N. Yu, C. Hollnagel, A. Blickenstorfer, S. S. Kollias, and R. Riener, "Comparison of MRI-compatible mechatronic systems with hydrodynamic and pneumatic actuation," IEEE/ASME Transactions on Mechatronics, vol. 13, no. 3, pp. 268-277, 2008.

[9] J. Wang, L. Yang, X. Luo, S. Mangan, and J. W. Derby, "Mathematical modeling study of scroll air motors and energy efficiency analysis part i," IEEE/ASME Transactions on Mechatronics, vol. 16, no. 1, pp. 112-121, 2011.
[10] F. Abry, X. Brun, S. Sesmat, and E. Bideaux, "Non-linear position control of a pneumatic actuator with closed-loop stiffness and damping tuning," in Proc. of the European Control Conference 2013, 2013.

[11] X. Shen and M. Goldfarb, "Simultaneous force and stiffness control of a pneumatic actuator," Journal of Dynamic Systems, Measurement, and Control, vol. 129, no. 4, pp. 425-434, 2007.

[12] A. Girin, F. Plestan, X. Brun, and A. Glumineau, "High-order slidingmode controllers of an electropneumatic actuator: Application to an aeronautic benchmark," International Journal of Control, vol. 79, no. 2, pp. 119-131, 2006.

[13] K. Xing, J. Huang, Y. Wang, J. Wu, Q. Xu, and J. He, "Tracking control of pneumatic artificial muscle actuators based on sliding mode and non-linear disturbance observer," IET Control Theory and Applications, vol. 4, no. 10, pp. 2058-2070, 2010.

[14] S.-E. Song, N. Cho, G. Fischer, N. Hata, C. Tempany, G. Fichtinger, and I. Iordachita, "Development of a pneumatic robot for mri-guided transperineal prostate biopsy and brachytherapy: New approaches," in Robotics and Automation (ICRA), 2010 IEEE International Conference on, 2010, pp. 2580-2585.

[15] C. Ying, Z. Jia-fan, Y. Can-jun, and N. Bin, "Design and hybrid control of the pneumatic force-feedback systems for arm-exoskeleton by using on/off valve," Mechatroncs, vol. 17, no. 6, pp. 325-335, 2007.

[16] D. Ben-Dov and S. Salcudean, "A force-controlled pneumatic actuator," IEEE Transactions on Robotics and Automation, vol. 11, no. 6, pp. 906911, 1995.

[17] R. van Varseveld and G. Bone, "Accurate position control of a pneumatic actuator using on/off solenoid valves," IEEE Transactions on Mechatronics, vol. 2, no. 6, pp. 195-204, 1997.

[18] M. Takaiwa and T. Noritsugu, "Application of pneumatic parallel manipulator as haptic human interface," in Proceedings of the IEEE/ASME International Conference on Advanced Intelligent Mechatronics, California, Sept 1999, pp. 185-190.

[19] X. Shen and M. Goldfarb, "On the enhanced passivity of pneumatically actuated impedence-type haptic interfaces," IEEE Transactions on Robotics, vol. 22, no. 3, pp. 470-480, 2006.

[20] A. Messina, N. I. Giannoccaro, and A. Gentile, "Experimenting and modelling the dynamics of pneumatic actuators controlled by the pulse width modulation (PWM) technique," Mechatronics, vol. 15, no. 7, pp. 859-881, 2005.

[21] K. Ahn and S. Yokota, "Intelligent switching control of pneumatic actuator using on/off solenoid valves," Mechatronics, vol. 15, no. 6, pp. 683-702, 2005.

[22] M.-C. Shih and M.-A. Ma, "Position control of a pneumatic cylinder using fuzzy PWM control method," Mechatronics, vol. 8, no. 3, pp. 241-253, 1998 .

[23] M. Taghizadeh, A. Ghaffari, and F. Najafi, "Improving dynamic performances of PWM-driven servo-pneumatic systems via a novel pneumatic circuit," ISA Transactions, vol. 48, no. 4, pp. 512-518, 2009.

[24] T. Nguyen, J. Leavitt, F. Jabbari, and J. E. Bobrow, "Accurate slidingmode control of pneumatic systems using low-cost solenoid valves," IEEE/ASME Transactions on Mechatronics, vol. 12, no. 2, pp. 216-219, 2007.

[25] H. Langjord and T. A. Johansen, "Dual-mode switched control of an electropneumatic clutch actuator," IEEE/ASME Transactions on Mechatronics, vol. 15, no. 6, pp. 969-981, 2010.

[26] V. I. Utkin, "Sliding mode control design principles and applications to electric drives," IEEE Transactions on Industrial Electronics, vol. 40, no. 1, pp. 23-36, 1993.

[27] V. I. Utkin and H.-C. Chang, "Sliding mode control on electromechanical systems," Mathematical Problems in Engineering, vol. 8, no. 4-5, pp. 451-473, 2002.

[28] X. Shen, J. Zhang, E. J. Barth, and M. Goldfarb, "Nonlinear model-based control of pulse width modulated pneumatic servo systems," Journal of Dynamic Systems, Measurement, and Control, vol. 128, pp. 663-669, 2006.

[29] S. Hodgson, M. Q. Le, M. Tavakoli, and M. T. Pham, "Sliding-mode control of nonlinear discrete-input pneumatic actuators," in Proceedings of IEEE/RSJ International Conference on Intelligent Robots and Systems (IROS), San Francisco, USA, September 2011, pp. 738-743.

[30] — , "Improved tracking and switching performance of an electropneumatic positioning system," Mechatronics, vol. 22, no. 1, pp. $1-$ $12,2012$.

[31] R. Moreau, M. T. Pham, M. Tavakoli, M. Q. Le, and T. Redarce, "Sliding-mode bilateral teleoperation control design for master-slave pneumatic servo systems," Control Engineering Practice, 2012. 